\title{
Gold Standard in Dental Photography: Digital Camera for Professional Dental Photography, EyeSpecial C-III
}

While visiting the dental exhibition in Cologne, IDS 2017, I tried to explore as many stands as possible belonging to the major equipment and material manufacturers present. We also went to the stand of Shofu Dental Corporation, the well-known producer coming from the "Land of the Rising Sun".

There, besides abrasives, diamonds \& carbids, cements, dental materials for both laboratories and dentists, we noticed the new digital camera for professional dental photography, EyeSpecial C-III. Because there were many practitioners asking for information about it, I gave up and continued my journey to collect information.

This year, during the FDI World Dental Congress 2018 in Buenos Aires, 5-8 September, I managed to hold this digital camera in my hand, to gather information and now I have the pleasure to introduce it to you.

In our current practice, clinicians and team members tend to circumvent the moment of taking pictures when they think about pulling out and holding up a big and heavy camera, finding help, selecting its functions, and hoping the captured images will be of high quality.

Shofu Dental Corporation (Kyoto 605-0983, Japan, http://www.shofu.co.jp) designed the camera EyeSpecial C-III especially for dentistry, as it is used for dental photography, case presentations in orthodontics and also in the dental laboratory.[1]

Designed for dentistry, this digital camera[1] [2] has a number of qualities:

- easy to use, so that anyone can capture high quality images;

- preset shooting modes, allowing faster and more reproducible images for a wide range of applications;

- image quality, allows exceptional photos to be taken without initial preparation;

- overall satisfaction, provides excellent service, saves user time.

\section{Easy to use}

The camera offers one-touch intuitive operation and a large LCD touch screen for viewing and scrolling, even when wearing gloves. Easy to handle, independent photo-taking, low shooting time, water and chemical resistant, easy disinfection, weighing only $590 \mathrm{~g}$, ergonomic design, easy to hold with only one hand these are all qualities that make the camera user-friendly and easy to use.

\section{Preset shooting modes}

EyeSpecial C-III is ideal for treatment planning, case documentation, patient education, case presentation and collaboration with the dental laboratory. The camera is equipped with eight preset shooting modes (Standard Surgery, Mirror, Face, Low Glare, Whitening, Tele Macro, Isolate Shade) to make images easier, faster and more reproducible for a wide range of applications.

\section{Image quality}

The camera offers twelve megapixels, an exceptional field depth, a $49 \mathrm{~mm}$ lens, fast autofocus, a proprietary FlashMatic system (4 LEDs around the lens and 4 side flashes) and an anti-shock mode, allow exceptional photos to be taken without photographic preparation previous. With Wi-Fi access, images can be instantly uploaded to a tablet, computer, or smartphone. It provides quality photos in compliance with OSHA and HIPPA protocols.

\section{Overall satisfaction}

The EyeSpecial C-III is a durable, easy-to-use product that delivers consistent results, preprogrammed modes make life easier and saves user time.

In conclusion, I recommend that you use the EyeSpecial C-III, a digital camera designed for professional dental photography, with which any member of the dental team without any photographic training can contribute to the high-quality photographic documentation of all activities in daily practice.

\section{References}

1. http://www.shofu.com/en/wp-content/uploads/sites/2/2014/05/ EyeSpecial-C-III-Brochure.pdf

2. http://www.thedentistryshow.co.uk/press-releases/eyespecial-c-iiiby-shofu

Florin - Eugen Constantinescu DMD, PhD Student

Editorial Director, Product News

DOI: 10.25241/stomaeduj.2018.5(3).prodnews.1

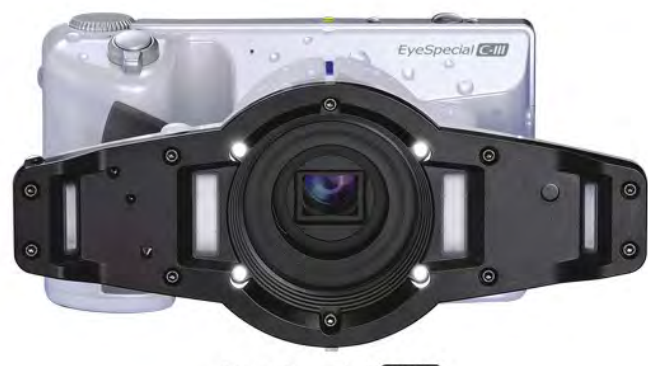

EyeSpecial C-III

Digital Camera for Professional Dental Photography EyeSpecial C-III Shofu Dental Corporation (Kyoto 605-0983, Japan) http://www.shofu.co.jp 